Revista de Metalurgia 50(3)

Julio-Septiembre 2014, e020

ISSN-L: 0034-8570

doi: http://dx.doi.org/10.3989/revmetalm.020

\title{
Estudio cinético de las reacciones de recocido en aleaciones de $\mathrm{Cu}-\mathrm{Ni}-\mathrm{Fe}$
}

\author{
Eduardo Donoso \\ Universidad de Chile, Facultad de Ciencias Físicas y Matemáticas, Departamento de Ciencia \\ de los Materiales, Avda. Tupper 2069, Santiago de Chile, Chile \\ Autor para la correspondencia: edonoso@ing.uchile.cl
}

Enviado: 19 Septiembre 2013; Aceptado: 20 Abril 2014; Publicado on line: 28 August 2014

\begin{abstract}
RESUMEN: Mediante Calorimetría Diferencial de Barrido (DSC) y medidas de microdureza Vickers se ha estudiado el comportamiento durante el recocido de las aleaciones $\mathrm{Cu}-45 \mathrm{Ni}-4 \mathrm{Fe}, \mathrm{Cu}-34 \mathrm{Ni}-11 \mathrm{Fe}$ y $\mathrm{Cu}-33 \mathrm{Ni}-22 \mathrm{Fe}$ templadas desde $1173 \mathrm{~K}$. El análisis de las curvas DSC, desde temperatura ambiente hasta los $950 \mathrm{~K}$, muestran la presencia de una reacción exotérmica asociada a la formación de la fase $\mathrm{FeNi}_{3}$ que nuclea a partir de una estructura modulada, y una reacción endotérmica que correspondería a la disolución de esta fase. Los parámetros cinéticos se calcularon a partir de la ecuación usual de Avrami-Erofeev, Kissinger modificado y funciones cinéticas integradas. Medidas de microdureza Vickers corroboraron la formación y disolución de fase $\mathrm{FeNi}_{3}$.
\end{abstract}

PALABRAS CLAVES: Aleaciones Cu-Ni-Fe; Calorimetría; Cinética; Endurecimiento

Citation / Cómo citar este artículo: Donoso, E. (2014) "Estudio cinético de las reacciones de recocido en aleaciones de Cu-Ni-Fe". Rev. Metal. 50(3): e020. doi: http://dx.doi.org/10.3989/revmetalm.020.

\begin{abstract}
Kinetic study of the annealing reactions in $\mathrm{Cu}-\mathrm{Ni}$-Fe alloys. The thermal aging of a $\mathrm{Cu}-45 \mathrm{Ni}-4 \mathrm{Fe}$, $\mathrm{Cu}-34 \mathrm{Ni}-11 \mathrm{Fe}$ and $\mathrm{Cu}-33 \mathrm{Ni}-22 \mathrm{Fe}$ alloys tempered from $1173 \mathrm{~K}$ have been studied from Differential Scanning Calorimetry (DSC) and microhardness measurements. The analysis of DSC curves, from room temperature to $950 \mathrm{~K}$, shows the presence of one exothermic reaction associated to the formation of $\mathrm{FeNi}_{3}$ phase nucleating from a modulate structure, and one endothermic peak attributed to dissolution of this phase. Kinetic parameters were obtained using the usual Avrami-Erofeev equation, modified Kissinger method and integrated kinetic functions. Microhardness measurements confirmed the formation and dissolution of the $\mathrm{FeNi}_{3}$ phase.
\end{abstract}

KEYWORDS: Cu-Ni-Fe alloys; Calorimetry; Hardening; Kinetic

Copyright: (C) 2014 CSIC. This is an open-access article distributed under the terms of the Creative Commons Attribution-Non Commercial (by-nc) Spain 3.0 License.

\section{INTRODUCCIÓN}

Debido a su notable resistencia a la corrosión las aleaciones cuproníqueles han sido ampliamente utilizadas en la industria química y naval. Sin embargo, el cobre y el níquel forman solamente soluciones sólidas. Muchas de las investigaciones a baja temperatura en las aleaciones de $\mathrm{Cu}-\mathrm{Ni}$ se realizaron suponiendo la existencia de una sola fase sólida homogénea de estructura cúbica de caras centradas. Para mejorar la resistencia mecánica de esta aleación se han utilizado mecanismos de endurecimiento tales como solución sólida sustitucional, procesado en frío y/o disminución del tamaño 
de grano (Nakanishi et al., 1974 y Shevakin et al., 1997). Por lo tanto, el efecto de endurecimiento por precipitación se puede lograr mediante la adición de un tercer componente, por ejemplo hierro.

De acuerdo al diagrama de fases ternario de $\mathrm{Cu}-\mathrm{Ni}-\mathrm{Fe}$ (Reghavan, 2004), a temperaturas menores que $820 \mathrm{~K}$, en la región cercana a concentraciones $\mathrm{Fe}-\mathrm{Ni}$, se encuentra una superred basada en el estequiométrico de la fase $\mathrm{FeNi}_{3}\left(\mathrm{~L}_{2}\right)$, estable, cúbico con parámetro de red de $1,08 \mathrm{~nm}$, la cual resulta ser tres veces la celda del cobre (o fase $\gamma$ ). A $723 \mathrm{~K}$, la fase $\mathrm{Ll}_{2}$ se encuentra en equilibrio con la fase $f c c$ desordenada, en dos diferentes rangos de composiciones $\left(\gamma_{1}\right.$ y $\left.\gamma_{2}\right)$. En aleaciones de $\mathrm{Cu}-30 \mathrm{Ni}-25 \mathrm{Fe}$, Li et al. (2001) encuentra que ocurre descomposición espinodal cuando el material es envejecido sobre $870 \mathrm{~K}$, con estructuras compuestas alternativamente de fases ricas en cobre y fases ricas en (Fe-Ni). Por otra parte, Servant et al., (2001), en aleaciones de $\mathrm{Cu}-17 \mathrm{Ni}-3 \mathrm{Fe}$, envejecidas a $773 \mathrm{~K}$ durante 2 horas, observó mediante Microscopía Electrónica de Transmisión (MET) precipitados de tamaño nanométrico en la matriz de cobre y en los bordes de grano. Sin embargo, López et al., (1996) encuentran que ocurre descomposición espinodal cuando se envejece $\mathrm{Cu}-46 \mathrm{Ni}-4 \mathrm{Fe}$ a $790 \mathrm{~K}$. En el presente trabajo se pretende obtener información adicional sobre el mecanismo de endurecimiento en aleaciones de $\mathrm{Cu}-\mathrm{Ni}-\mathrm{Fe}$, utilizando calorimetría diferencial de barrido (DSC) y medidas de microdureza Vickers.

\section{MATERIALES Y MÉTODOS}

Las aleaciones utilizadas se prepararon en un horno de inducción, en atmósfera inerte (Ar), a partir de cobre electrolítico (99,95\% de pureza), hierro y níquel de alta pureza. Los lingotes obtenidos se recocieron a $1173 \mathrm{~K}$ durante 24 horas (en atmósfera inerte) a fin de homogeneizarlos. Después de un análisis químico se encontró que sus composiciones correspondían a $\mathrm{Cu}: 44,9$ at.\%, Ni: 3,8 at.\% $\mathrm{Fe}$ (aleación $\mathrm{Cu}-45 \mathrm{Ni}-4 \mathrm{Fe}$ ), $\mathrm{Cu}: 33,9$ at. $\%$, Ni: 11,1 at. $\% \mathrm{Fe}$ (aleación $\mathrm{Cu}-34 \mathrm{Ni}-11 \mathrm{Fe}$ ) y $\mathrm{Cu}: 32,8$ at. $\%$, Ni: 22,3 at. $\%$ Fe (aleación Cu-33Ni-22Fe). Posteriormente, los materiales se laminaron en frío hasta $3 \mathrm{~mm}$ de espesor con recocidos intermedios de 1 hora a $1173 \mathrm{~K}$. Luego del último recocido, las aleaciones se templaron en agua.

Para el análisis calorimétrico se utilizó un equipo de Calorimetría Diferencial de Barrido (DSC) TA Instruments Q10. Los diagramas de DSC se obtuvieron a diversas velocidades de calentamiento $(\beta)$ $\left(\beta=0,083 ; 0,167 ; 0,333\right.$ y $\left.0,50 \mathrm{~K} \mathrm{~s}^{-1}\right)$, bajo un flujo de argón de $10^{-4} \mathrm{~m}^{3} \mathrm{~min}^{-1}$. A fin de aumentar la precisión de las medidas, se utilizó como referencia un disco de cobre de alta pureza recocido durante un largo período. La corrección de la línea base se llevó a cabo por el método previamente descrito en publicaciones anteriores (Varchavsky et al., 2002; Varchavsky et al., 2003; Donoso et al., 2007).

Las medidas de microdureza Vickers se efectuaron a temperatura ambiente en un microdurómetro Duramin -1/-2 Struers de alta precisión, aplicando una carga de $1,96 \mathrm{~N}$ durante $10 \mathrm{~s}$. Cada valor de microdureza corresponde al promedio de diez medidas, con una desviación estándar de aproximadamente un $2 \%$.

\section{RESULTADOS Y DISCUSIÓN}

La Figura 1 muestra curvas calorimétricas típicas para las tres aleaciones en estudio: (a) $\mathrm{Cu}-45 \mathrm{Ni}-4 \mathrm{Fe}$, (b) $\mathrm{Cu}-34 \mathrm{Ni}-11 \mathrm{Fe}$ y (c) $\mathrm{Cu}-33 \mathrm{Ni}-22 \mathrm{Fe}$, templadas desde $1173 \mathrm{~K}$, a la velocidad de calentamiento lineal $\beta=0,167 \mathrm{~K} \mathrm{~s}^{-1}$. Las curvas calorimétricas para las otras velocidades de calentamiento lineal tienen un comportamiento similar al mostrado en la Figura $1 \mathrm{y}$ por lo tanto no se recogen en este trabajo. Las curvas DSC muestran, en el rango de temperaturas estudiado, en cada aleación, una reacción exotérmica (picos 1, 3 y 5) y a mayores temperaturas una reacción endotérmica (picos 2, 4 y 6). De acuerdo a los antecedentes establecidos en la literatura (López et al., 1996; Li et al., 2001; Servant, 2001; Reghavan, 2004), los picos exotérmicos corresponden a la reacción de formación de la fase estable $\mathrm{FeNi}_{3}\left(\mathrm{L1}_{2}\right)$ y los endotérmicos a la disolución de la fase anterior.

En la Figura 2 se muestran, para las tres aleaciones en estudio (etapas 1 a 6 ), los valores de las temperaturas del máximo de los picos $\mathrm{T}_{\mathrm{p}}$ (temperatura a la cual la velocidad de reacción es máxima) en función de la velocidad de calentamiento lineal $\beta$. El hecho de que las temperaturas de los picos de las curvas calorimétricas se desplacen a temperaturas más altas al aumentar la velocidad de calentamiento

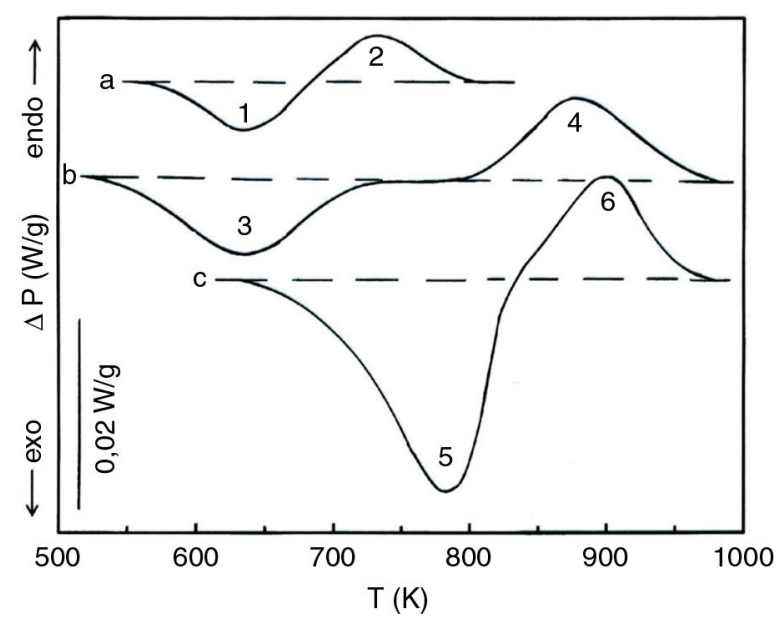

Figura 1. Curvas DSC de las aleaciones en estudio templadas desde $1173 \mathrm{~K}, \beta=0,167 \mathrm{Ks}^{-1}$; (a) $\mathrm{Cu}-45 \mathrm{Ni}-4 \mathrm{Fe}$; (b) $\mathrm{Cu}-34 \mathrm{Ni}-11 \mathrm{Fe}$ y (c) $\mathrm{Cu}-33 \mathrm{Ni}-22 \mathrm{Fe}$. 


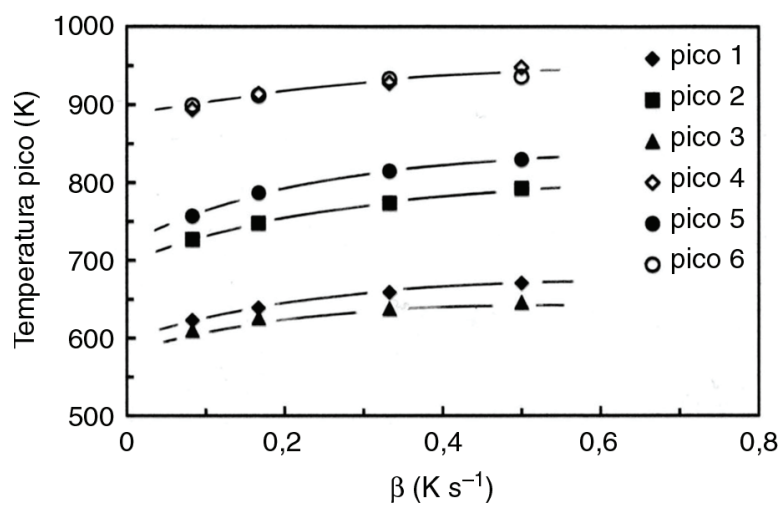

Figura 2. Temperatura de los picos en función de la velocidad de calentamiento para todas las etapas observadas.

pone de manifiesto el control cinético del proceso (Varschavsky et al., 1991).

Con objeto de analizar la posible relación existente entre los procesos observados y las propiedades mecánicas de las tres aleaciones estudiadas (Cu-45Ni-4Fe, Cu-34Ni-11Fe y Cu-33Ni-22Fe), se han realizado medidas de microdureza Vickers en muestras calentadas en el calorímetro a una velocidad de $0,167 \mathrm{~K} \mathrm{~s}^{-1}$ y luego extraídas a temperaturas seleccionadas de los diagramas de DSC registrados para las tres aleaciones. Las temperaturas a las que se han extraído las muestras del aparato de DSC corresponden a la temperatura ambiente (material templado) y a las temperaturas cercanas de los picos exotérmicos y endotérmicos. Tanto las temperaturas seleccionadas como la microdureza Vickers determinadas para las mismas se muestran en la Tabla 1. Los resultados ponen de manifiesto, en el caso del material templado (sin tratamiento de envejecimiento) un aumento de la dureza con el incremento de la concentración de Fe, debido principalmente al cambio de parámetro de red de la aleación (disminución del parámetro de red de la aleación cuando aumenta la concentración de $\mathrm{Ni}$ e

TABLA 1. Microdurezas Vickers a temperatura ambiente en función de la temperatura de recocido

\begin{tabular}{lcc}
\hline Aleación & T (K) & Hv \\
\hline Cu-45Ni-4Fe & 300 & 140 \\
& 650 & 162 \\
$\mathrm{Cu}-34 \mathrm{Ni}-11 \mathrm{Fe}$ & 770 & 146 \\
& 300 & 156 \\
& 650 & 170 \\
$\mathrm{Cu}-33 \mathrm{Ni}-22 \mathrm{Fe}$ & 770 & 175 \\
& 950 & 158 \\
& 300 & 168 \\
& 770 & 182 \\
& 950 & 173 \\
\hline
\end{tabular}

incremento de dicho parámetro cuando aumenta la concentración de Fe (Pearson, 1958). También, se puede observar, en las tres aleaciones, un notable incremento de la dureza asociado a la aparición de los picos exotérmicos, por el contrario, se observa una gran disminución de la dureza asociado a las reacciones endotérmicas. El incremento de la dureza podría atribuirse a una descomposición espinodal y a la formación de una fase $\mathrm{FeNi}_{3}$. La posterior disminución de la dureza se asocia a la disolución de dichas fases.

El análisis cinético de la reacción exotérmica de formación de $\mathrm{FeNi}_{3}$, se realizó utilizando la ecuación usual de Avrami-Erofeev (Donoso et al., 2012a; Donoso et al., 2012b; López et al., 2014), empleada para reacciones heterogéneas, bajo condiciones no isotermas:

$$
\alpha=1-\exp \left[-(A \theta)^{n}\right]
$$

donde $\alpha$ es la fracción reaccionada a la temperatura $T, A$ es el factor pre-exponencial de Arrhenius y $\theta$ es el tiempo reducido e igual a $R T^{2} / \beta E \exp (-E / R T)$, siendo $E$ la energía de activación, $\beta$ la velocidad de calentamiento lineal y $R$ la constante de los gases (Ozawa, 1976). El análisis cinético de las curvas calorimétricas mostradas en la Figura 1, se ha realizado considerando que la fracción de reacción, para un tiempo determinado, se puede expresar mediante la ecuación (2):

$$
\frac{d \alpha}{d t}=\frac{1}{S} \frac{d \alpha_{t}}{d t}
$$

donde $a_{t}$ es el área bajo la curva al tiempo $t$ y $S$ es el área total bajo la curva.

De la integración de la ecuación anterior se tiene que la fracción de reacción $\alpha$ al tiempo $t$ está dada por la expresión: $\alpha=a_{t} / S$ (Donoso, 2010).

Las energías de activación de las reacciones químicas puestas de manifiesto en el estudio mediante calorimetría, se calcularon a partir de la ecuación de Kissinger modificada (Ec. 3) (Mittemeijer et al., 1988):

$$
\ln \left(\frac{T_{p}^{2}}{\beta}\right)=\frac{E}{R T_{p}}+\ln \left(\frac{E}{A R}\right)
$$

donde $T_{p}$ es la temperatura del máximo del pico. $E \mathrm{y}$ $A$ se calcularon a partir de la representación gráfica de $\ln \left(T_{p}^{2} / \beta\right.$ ) en función de $1 / T_{p}$ (Fig. 3). La ecuación de Kissinger utilizada es un método que permite calcular la energía de activación sin conocimiento previo ni asunción del modelo cinético. Hay autores que han puesto en duda la validez del mismo pues no es claramente un método isoconversional. 


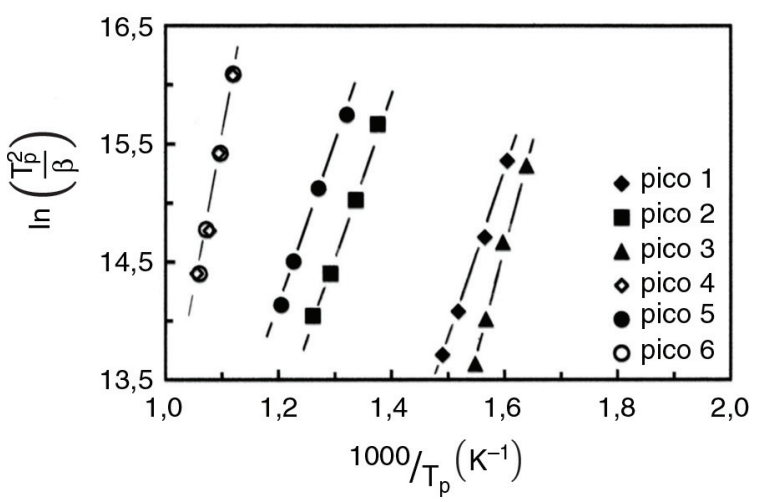

Figura 3. Gráfico de Kissinger utilizado en la determinación de las energías de activación y factores pre-exponenciales.

Sin embargo, estudios recientes han puesto de manifiesto que en un calentamiento lineal, los valores de $\alpha$ máximo son dependientes del modelo, pero prácticamente independientes de la velocidad de calentamiento, por lo que para efectos prácticos es un procedimiento isoconversional (Sánchez-Jiménez et al., 2008).

Los valores obtenidos de $E$ y constante $A$, se muestran en la Tabla 2. Los valores de las energías de activación obtenidos para los picos 1,3 y 5 (reacciones exotérmicas) resultaron más bajos que las energías de difusión de $\mathrm{Ni}$ en $\mathrm{Cu}\left(204 \mathrm{~kJ}^{-1} \mathrm{~mol}^{-1}\right.$ (Brown et al., 1980)) y Fe en $\mathrm{Cu}\left(218 \mathrm{~kJ} \mathrm{~mol}^{-1}\right.$ (Brown et al., 1980)), lo cual puede ser atribuido a la fuerte contribución de las vacantes introducidas durante el temple. Por otra parte, los valores de $E$ para los picos 2, 4 y 6 (reacciones endotérmicas) resultaron similares a las energías de difusión de los solutos en la matriz de cobre. Como es de esperar, la energía de activación para la aleación más diluida en Fe es más baja que aquéllas que tienen mayor concentración de Fe.

A partir de gráficos $\ln \ln [1 /(1-\alpha)]$ versus $\ln (\theta)$, utilizando la ecuación (1) y valores de $\alpha$ obtenidos experimentalmente a partir de las curvas calorimétricas (con valores de $\alpha$ comprendidos entre $0,1 \mathrm{y}$ $0,9)$, se evaluó la constante $n$ de la pendiente de las curvas, cuyos valores se muestran en la Tabla 2 . De acuerdo a la literatura (Pérez-Maqueda et al., 2003),

TABla 2. Parámetros cinéticos de las reacciones químicas producidas en el calentamiento no isotérmico de las aleaciones

\begin{tabular}{lccc}
\hline & $\mathbf{E}\left(\mathbf{k J} \mathbf{~ m o l}^{-\mathbf{1}}\right)$ & $\mathbf{A}\left(\mathbf{s}^{-\mathbf{1}}\right)$ & $\mathbf{n}$ \\
\hline pico 1 & 118 & $3 \times 10^{7}$ & 1,10 \\
pico 2 & 187 & $6 \times 10^{6}$ & - \\
pico 3 & 155 & $7 \times 10^{10}$ & 1,16 \\
pico 4 & 210 & $2 \times 10^{10}$ & - \\
pico 5 & 148 & $3 \times 10^{10}$ & 1,14 \\
pico 6 & 214 & $8 \times 10^{10}$ & - \\
\hline
\end{tabular}

los valores cercanos a la unidad pueden explicarse de dos maneras: a) nucleación instantánea y crecimiento unidimensional de los núcleos controlados por procesos de interface, o b) nucleación instantánea y crecimiento bidireccional de los núcleos controlados por procesos de difusión.

El análisis cinético de las reacciones endotérmicas (picos 2, 4 y 6) fue realizado sobre la base de la teoría de Varschavsky et al., 2002, que controla la cinética de disolución de precipitados y es adecuada para utilizarse en condiciones anisotermales y que se representa mediante la ecuación (4):

$$
F(\alpha)=1-(1-\alpha)^{\frac{2}{3}}=A_{1}^{*} \theta
$$

Esta ecuación, describe situaciones tridimensionales con simetría esférica controladas por difusión, mientras que la ecuación (5) describe mejor situaciones que involucran difusión unidimensional con geometría de placas. En ambos casos, $A_{1}^{*}$ y $A_{2}^{*}$ son constantes y $\theta$ es el tiempo reducido definido anteriormente.

$$
F(\alpha)=\left[1(1-\alpha)^{1 / 3}\right]^{2}=A_{2}^{*} \theta
$$

La representación gráfica de los valores $\mathrm{F}(\alpha)$ en función de $\theta$, para la aleación $\mathrm{Cu}-34 \mathrm{Ni}-11 \mathrm{Fe}$ a $\beta=0,167 \mathrm{~K} \mathrm{~s}^{-1}$, se muestra en la Figura 4. En ella se puede observar claramente que el modelo para situaciones tridimensionales con simetría esférica describe mucho mejor el comportamiento de la reacción endotérmica. Las curvas para las otras aleaciones tienen un comportamiento similar al mostrado en la Figura 4 y no se recogen en este trabajo.

De los gráficos $F(\alpha)$ vs. $\theta$, a partir de la ecuación (4), se obtienen los valores de $A_{1}^{*}$ iguales a $4,4 \times 10^{6}$; $0,9 \times 10^{10}$ y $6 \times 10^{10} \mathrm{~s}^{-1}$ para los picos 2,4 y 6 respectivamente. Se observa claramente que el comportamiento de la disolución es ajustable al modelo cinético de la ecuación (4) utilizado para las tres

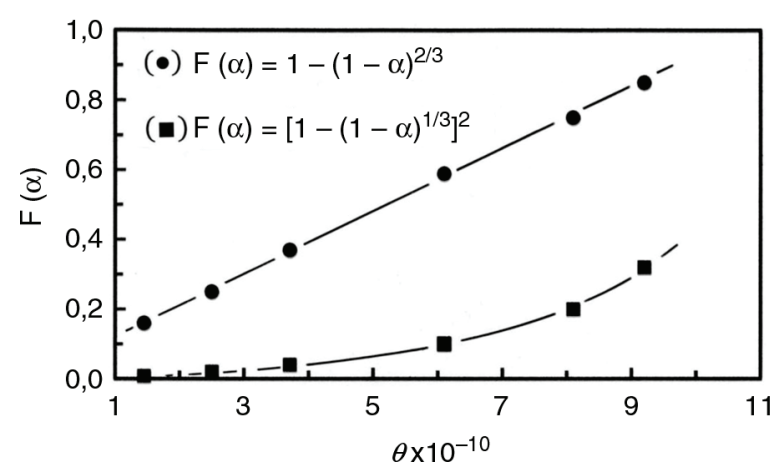

FigurA 4. Gráficos de las funciones cinéticas integradas en función de $\theta$ para la aleación $\mathrm{Cu}-34 \mathrm{Ni}-11 \mathrm{Fe}$. 
aleaciones en estudio, ya que resultaron ser rectas de pendientes $A_{1}^{*}$. Además, se puede constatar que estos valores de $A_{1}^{*}$ son del mismo orden de magnitud que los valores de $A$ obtenidos a partir del modelo de Kissinger modificado.

\section{CONCLUSIONES}

El análisis cinético y calorimétrico de los diagramas de DSC obtenidos a diferentes velocidades de calentamiento junto a las determinaciones de microdureza realizadas, permiten concluir que el recocido de muestras templadas de las aleaciones de $\mathrm{Cu}-45 \mathrm{Ni}-4 \mathrm{Fe}, \mathrm{Cu}-34 \mathrm{Ni}-11 \mathrm{Fe}$ y $\mathrm{Cu}-33 \mathrm{Ni}-22 \mathrm{Fe}$ induce su endurecimiento por precipitación de fases de composición $\mathrm{FeNi}_{3}$. Asimismo, se puede concluir que la función cinética integrada $F(\alpha)=1-(1-\alpha)^{2 / 3}$ es la que mejor describe el mecanismo de disolución de las partículas de $\mathrm{FeNi}_{3}$.

\section{AGRADECIMIENTOS}

El autor desea agradecer al Fondo Nacional de Desarrollo Científico y Tecnológico, FONDECYT, Proyecto $\mathrm{N}^{\mathrm{o}} 1110007$, por el apoyo financiero y a la Universidad de Chile, Facultad de Ciencias Físicas y Matemáticas, Departamento de Ciencia de los Materiales, por la facilidades otorgadas para desarollar esta investigación.

\section{REFERENCIAS}

Brown, A.M., Ashby, M.F. (1980). Correlation for diffusion constants. Acta Metall. 28 (8), 1085-1101. http://dx.doi. org/10.1016/0001-6160(80)90092-9.

Donoso, E., Diánez, M.J., Sayagués, M.J., Criado, J.M., Varschavsky, A., Díaz, G. (2007). Non isothermal calorimetric study of the precipitation processes in a $\mathrm{Cu}-10 \%$ Ni-3\% Al. Rev. Metal. 43 (2), 117-124. http://dx.doi. org/10.3989/revmetalm.2007.v43.i2.

Donoso, E. (2010). Influence of cobalt and chromium additions on the precipitation processes in a $\mathrm{Cu}-4 \mathrm{Ti}$ alloys. Rev. Metal. 46 (6), 542-547. http://dx.doi.org/10.3989/ revmetalmadrid.1042.

Donoso, E., Espinoza, R., Diánez, M.J., Criado, J.M. (2012a). Microcalorimetric study of the annealing hardening mechanism of a Cu-2.8Ni-1.4Si (at.\%) alloy. Mater. Sci. Eng. A 556, 612-616. http://dx.doi.org/10.1016/j.msea.2012.07.035.

Donoso, E., Diánez, M.J., Criado, J.M. (2012b). Non-isothermal microcalorimetric evaluations in quenched and cold-rolled
Cu-9Ni-5.5Sn alloys. Rev. Metal. 48 (1), 67-75. http:// dx.doi.org/10.3989/revmetalm.1136.

Li, H.X., Hao, X.J., Zhao, G., Hao, S.M. (2001). Characteristics of the continuous coarsening decomposed $\mathrm{Cu}-$ Ni-Fe alloy. J. Mater. Sci. 36 (3), 779-784. http://dx.doi. org/10.1023/A:1004865714352.

López Hirata, V.M., Hirano, K.I. (1996). A study of spinodal decomposition in $\mathrm{Ni}-30$ at. $\% \mathrm{Cu}$ and $\mathrm{Cu}-46$ at. $\% \mathrm{Ni}-4$ at. $\% \mathrm{Fe}$ alloys using electrical resistivity measurements. $J$. Mater. Sci. 31 (7), 1703-1706. http://dx.doi.org/10.1007/ BF00372181.

López, F.A., Sierra, M.J., Rodríguez, O., Millán, R., Alguacil, F.J. (2014). Non-isothermal kinetics of the thermal desorption of mercury from a contaminated soil. Rev. Metal. 50 (1), e001. http://dx.doi.org/10.3989/revmetalm.001.

Mittemeijer, E.J., Cheng, L., Van der Shaaf, P.J., Brakmany, C.M., Korevaar, B.M. (1988). Analysis of nonisothermal transformation kinetics; tempering of iron-carbon and iron- nitrogen martensites. Metall. Trans. A 19 (4), 925-932. http:// dx.doi.org/10.1007/BF02628377.

Nakanishi, K., Suzuki, H. (1974). Analysis of the grain size dependence of the yield stress in copper-aluminum and copper-nickel alloys. T. Jpn. I. Met. (JIM) 15 (6), 435-440.

Ozawa, T. (1976). A modified method for kinetic analysis of thermoanalytical data. J. Thermal Anal. 9 (3), 369-373. http://dx.doi.org/10.1007/BF01909401.

Pearson, W.B. (1958). A Handbook of Lattice Spacings and Structures of Metals and Alloys, Pergamon Press, pp. 571-574.

Pérez-Maqueda, L.A., Criado, J.M., Málek, J. (2003). Combined kinetic analysis for crystallization kinetics of noncrystalline solids. J. Non-Cryst. Solids 320 (1-3), 84-91. http://dx.doi.org/10.1016/S0022-3093(03)00023-1.

Reghavan, V. (2004). Cu-Fe-Ni (Copper-Iron-Nickel). J. Phase Eq. Diff. 25 (6), 547-549. http://dx.doi.org/10.1007/s11669004-0074-0.

Sánchez-Jiménez, P.E., Criado, J.M., Pérez-Maqueda, L.A. (2008). Kissinger kinetic analysis of data obtained under different heating schedules. J. Thermal Anal. Cal. 94 (2), 427-432. http://dx.doi.org/10.1007/s10973-008-9200-2.

Servant, C., Guymont, M., Lyon, O. (2001). A new phase in the ternary system Cu-Fe-Ni. Scripta Mater. 45 (1), 103-108. http://dx.doi.org/10.1016/S1359-6462(01)00998-8.

Shevakin, J.F., Tsypin, M.J. (1997). The curves of plastic flow and deformation strengthening of some solid solution on the basis of copper. Adv. Perfomance Mater. 4 (2), 233237. http://dx.doi.org/10.1023/A:1008655832363.

Varschavsky, A., Donoso, E. (1991). Short-range ordering by excess and thermal vacancies during linear heating experiments in $\alpha-\mathrm{Cu}-\mathrm{Al}$ alloys. Mater. Sci. Eng. A 145 (1), 95-107, http://dx.doi.org/10.1016/0921-5093(91)90299-3.

Varschavsky, A., Donoso, E. (2002). Energetic and kinetic evaluations conducted in a quasi-binary $\mathrm{Cu}-1$ at. $\% \mathrm{Co}_{2} \mathrm{Si}$ alloy through DSC. J. Termal Anal. Cal. 68 (1), 231-241. http:// dx.doi.org/10.1023/A:1014969618372.

Varschavsky, A., Donoso, E. (2003). Energetic and kinetic evaluations in a quasi-binary $\mathrm{Cu}-1$ at. $\% \mathrm{Co}_{2} \mathrm{Si}$. Mater. Lett. 57 (7), 1266-1271. http://dx.doi.org/10.1016/S0167-577X (02)00970-9. 